\title{
Socialized Multi-Agent System Rendezvous via Networks of Networks
}

\author{
Yunzhong Song \\ College of Electrical Engineering and Automation, Henan Polytechnic University, 2001 Century Avenue, Jiaozuo \\ 454003, P.R.China \\ Ziyi Fu \\ College of Electrical Engineering and Automation, Henan Polytechnic University, 2001 Century Avenue, Jiaozuo \\ 454003, P.R.China \\ Fuzhong Wang \\ College of Electrical Engineering and Automation, Henan Polytechnic University, 2001 Century Avenue, Jiaozuo \\ 454003, P.R.China
}

E-mail: songhpu@126.com,fzy@hpu.edu.cn,wangfzh@hpu.edu.cn

www.hpu.edu.cn

\begin{abstract}
Networks of networks paradigm was introduced to explore the rendezvous problems of leader-follower multi-agent system, where the rendezvous actors were categorized into the target object, the leader agents, the informed follower agents and the isolated follower agents, respectively. Then, three humanized system inspired strategies were investigated. Results demonstrated that, the democracy strategy could fulfill the rendezvous task in expenses with a long convergent time and the autarchy democracy could come to the targeted object quickly with the risk of failing in rendezvous, while the mixed strategy cared about both of the convergent speed and the utmost task, would take autarchy strategy when the connections were available for the follower agents, while the democracy rules had to be used if the connections were not possible.
\end{abstract}

Keywords: networks of networks, leader-follower multi-agent network, rendezvous problems, the democracy strategy, the autarchy strategy, the mixed strategy

\section{Introduction}

In recent years, the distributed control of multi-agent network has been paid more attentions from different disciplines. For instance, Ref. 1 analyzed the issue of how the ratio of leaders to followers affects connectivity. Ref. 2 discussed the consensus problems in undirected networks of agents with fixed topology and switching topology. In Ref. 3, the direct control laws were used in a heterogeneous network. Ref. 4 provided sufficient conditions for rendezvous in first order leader-follower network; Ref. 5 studied the distributed tracking control of the leader-follower directed network with noisy measurement. Ref. 6 investigated the consensus problems in leader-follower network with fixed topology and switching topology.

Although the consensus research of multi-agent networks and leader-follower multi-agent networks provided valuable results, there still have been some obvious limitations. For instance, the realization mechanism of the swarm has been considered rarely. 
But in actual society, the swarm members can interact with each other in various styles, for example, the followers may be kept in the group or be left alone according to the different utmost objectives; and as the same way, the leaders may also play with the followers in the unselfish ways or in the selfish ways, that can be boiled down to the costs and the capabilities of the leaders. Lately, the networks of networks, like Ref.7 which paid much more attention to the coupling among the networks, was much more powerful in modeling the real world systems, where interdependence of networks and the effect of this interdependence on the structural and functional behavior of the coupled system were considered into detail. So, it is necessary to be clarified that whether the networks of networks form mechanism can be verified feasible or not in leader-follower multiagent systems. The already existing results including the different layers built according to the different relationship among agents, for example, one layer can be used to express the relationship from the spatial location of the agents, that is, if they are near each other enough in spatial physical distance, they can have connections with each other; and the another layer can be assigned to express their social relationships, such as friendships, and classmates, that is if they are friends or have been classmates before, they can be categorized into one layer, and the connection strength can be determined with their intimateness. The style we deal with networks of networks here can enrich the results of networks of networks and it can be referred further. In this paper, the three strategies namely the democracy strategy, the autarchy strategy and the mixed strategy would be used in rendezvous problem of leader-follower network. Specific to leader-follower multi-agent network, the democracy strategy means that the motion of agent is affected by the other ones located within the sensing zone of the agent. And the autarchy strategy is such a strategy, in which the motion of leader is not affected by any agents, while follower only gets the influence of the initial nearest leader within its sensing zone. The mixed strategy is composed of the autarchy one and the democracy one. In the mixed strategy, all the leaders and some followers which are affected by leader initially take the autarchy strategy, while the other followers which are called isolated followers take the democracy strategy.

Here, we must note that in case of brevity and the limited space of the paper, only the democracy strategy is covered.

\section{Preliminaries}

Consider $\mathrm{N}$ agents evolving in $\mathrm{R}^{2}$. We use single integrator agents whose motions obey the model:

$\dot{x}_{i}^{j}=u_{i}^{j}, i \in \mathrm{N}=\{1, \ldots, N\}, j \in \mathrm{N}=\{1, \ldots, M\}$,
Where the subscript denotes the order number of the agents in the corresponding layer, whereas the superscript denotes the order number of the layer that agents belong to. So, here in (1), N and M denote the biggest order number of intra-layer and inter-layer. And the rest can be done in the same manner. In case of convenience, the node order number and its corresponding expression in networks of networks style is listed in Table 1.

Table1. The node order number and its corresponding expression in networks of networks style.

\begin{tabular}{cccccccccc}
\hline \multicolumn{1}{c}{ Followers } & \multicolumn{3}{c}{ Leaders } & Target \\
\hline V1 & V2 & V3 & V4 & V5 & V6 & V7 & V8 & V9 & V10 \\
$x_{1}^{3}$ & $x_{2}^{3}$ & $x_{3}^{3}$ & $x_{4}^{3}$ & $x_{1}^{4}$ & $x_{5}^{3}$ & $x_{1}^{2}$ & $x_{2}^{2}$ & $x_{3}^{2}$ & $x_{1}^{1}$ \\
\hline
\end{tabular}

Let $g=\{V, E\}$ describe the group topology, that consists of a set of vertices, $\mathrm{V}=\{1, \ldots, \mathrm{N}\}$, representing the team members, and a set of edges, $\mathrm{E}=\{(i, j) \in \mathrm{V} \times \mathrm{V} \mid$ $i \in \mathrm{N}\}$ representing the active inter-agent communication links. If $\mathrm{g}$ is an undirected graph, there is a line between node $i$ and $j$, representing the impact between them is mutual. If $\mathrm{g}$ is a directed graph, the line $(i, j)$ is directed, which consists of starting point $i$ and finishing point $j$, representing node $i$ makes an impact on node $j$.

According to the different functions of the agents in leader-follower network, the agents can be divided into two categories, i.e. leaders and followers. The leaders were capable of knowing the details of the global task that is to come to a given target, while the followers just need to keep in touch with leaders to reach the target. The agents belong either to the subset of leaders, $\mathrm{N}_{l}$, or to the subset of followers, $\mathrm{N}_{f}$, where $\mathrm{N}_{l} \cup \mathrm{N}_{f}=\mathrm{N}, \mathrm{N}_{l} \cap \mathrm{N}_{f}=\phi$ and the number of agents in each set is given by $\left|\mathrm{N}_{l}\right|=N_{l}$ and $\left|\mathrm{N}_{f}\right|=N_{f}$ respectively. Due to limited sensing capability of the sensors, each agent has a limited sensing zone of radius $\Delta>0$. At any given time, the set of agents located within the sensing zone of agent $i \in \mathrm{N}$ are referred to as the neighbours of agent $i$, $\mathrm{N}_{i}=\left\{j \in \mathrm{N}:\left|x_{i}-x_{j}\right| \leq \Delta\right\}$. Each agent has knowledge of the relative coordinates to its neighbouring agents, but cannot detect or communicate with agents outside its sensing zone. Note that both $\mathrm{g}$ and $\mathrm{N}_{i}$ are time-varying. The difference between leaders and followers is that leaders are aware of the location of the target and can control group but the followers cannot.

We start by introducing a notation for the distance between two arbitrary agents $i$ and $j$. Let $\delta_{i, j}^{k, l}=\delta_{j, i}^{l, k}=\left|x_{i}^{k}-x_{j}^{l}\right|=\sqrt{\left(x_{i}^{k}-x_{j}^{l}\right)^{T}\left(x_{i}^{k}-x_{j}^{l}\right)} \geq 0$ Since we are considering a physical system we can assume that $x_{k}(t)$ is a continuous function for any agent 
$k \in \mathrm{N}$. The time derivative $\dot{\delta}_{i j}$ is not directly defined when $\delta_{i, j}^{k, l}=0$ so here we shall instead consider the time derivative of $\left(\delta_{i, j}^{k, l}\right)^{2}$

$$
\frac{d\left(\delta_{i, j}^{k, l}\right)^{2}}{d t}=2 \delta_{i, j}^{k, l} \dot{\delta}_{i, j}^{k, l}=2\left(x_{i}^{k}-x_{j}^{l}\right)^{\mathrm{T}}\left(\dot{x}_{i}^{k}-\dot{x}_{j}^{l}\right)
$$

To describe the relationship between leaders and target, the target attraction function is defined as

$$
F\left(x_{j}^{l}, d\right)= \begin{cases}f\left(\delta_{j}^{l}\right) \frac{d-x_{j}^{l}}{\delta_{j}^{l}}, & \delta_{j}^{l}>0 \\ 0, & \delta_{j}^{l}=0 .\end{cases}
$$

where $d$ is the location of the target and define $\delta_{j}^{l}=\left|x_{j}^{l}-d\right|$ representing the agent $x_{j}^{l}$ 's distance to the target. At any given position $x_{j}^{l} \neq d$, the direction of $F\left(x_{j}^{l}, d\right)$ is towards the target and the magnitude is decided by the continuous scalar function $f\left(\delta_{j}^{l}\right) \geq 0$.

In the following analysis, the leader-follower multi-agent network that consists of four leaders and five followers is illustrated. The agents are tagged with numbers, starting at 1 at first, followed with 2, 3 and the other numbers, where agents from 1 to 5 belong to follower agents, whereas 6 to 9 belong to leader agents and node 10 is the target. Without loss of generality, we assume that the target is the origin of coordinates and $f\left(\delta_{j}\right)=\beta \delta_{j}, \beta=0.5$. The initial configuration of each agent can be seen in Tab.2.

Table 2. Initial values of the simulation work.

\begin{tabular}{ccccccccccc}
\hline \multicolumn{1}{c}{ Followers } & \multicolumn{1}{c}{ Leaders } & Target \\
\hline & 1 & 2 & 3 & 4 & 5 & 6 & 7 & 8 & 9 & 10 \\
$\mathrm{x}$ & 27.5 & 28 & 28 & 28 & 32 & 17 & 21.5 & 22 & 21 & 0 \\
$\mathrm{y}$ & 0 & -0.2 & -4 & 4 & -1 & -2 & -1 & 0.5 & 1 & 0 \\
\hline
\end{tabular}

\section{Results and Analysis}

In the democracy strategy, the motion of agent is affected by the other ones located within the sensing zone of the agent. If agent $\mathrm{i}$ has an impact on $\mathrm{j}$, agent $\mathrm{i}$ is also affected by $\mathrm{j}$. In other words, interactions among each agent are undirected. The topology of group is thus described by undirected graph $\mathrm{g} 1=\{\mathrm{V} 1, \mathrm{E} 1\}$.

The dynamics for an arbitrary follower agent $\mathrm{i}$ are given by

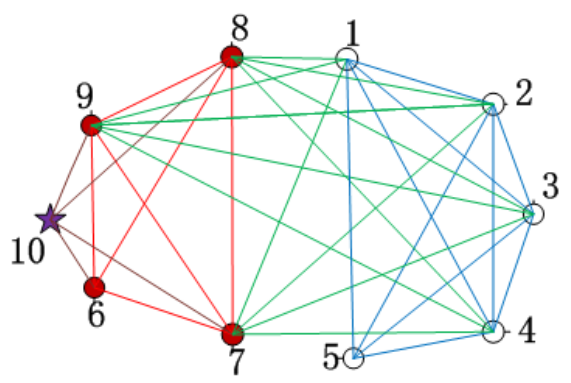

(a) Topological structure

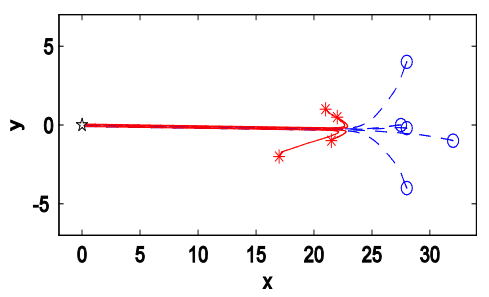

(b) Position rendezvous

Fig. 1. Simulation results in the democracy strategy.

$\dot{x}_{i}^{j}=-\sum_{k \in \mathrm{N}_{i}^{j}}\left(x_{i}^{j}-x_{k}^{l}\right)=-N_{i}^{j} x_{i}^{j}+\sum_{k \in \mathrm{N}_{i}^{j}} x_{k}^{l}$

Where $x_{i}^{j}$ denotes the $\mathrm{i}^{\text {th }}$ agent is in the $\mathrm{j}^{\text {th }}$ layer, and $x_{k}^{l}$ denotes the $\mathrm{k}^{\text {th }}$ neighbor of $x_{i}^{j}$, which is in the $1^{\text {th }}$ layer of the networks. We can conclude that the motions of followers are decided by their neighbors. Considering the situation of two dimensions, the dynamics can be looked as the resultant vectors of themselves and their neighbors, where the directions of all the agents are the same corresponding to the resultant vectors, and the speeds of the swarm motion members can also be decided by the norm of the resultant vectors. For an arbitrary leader agent $j \in \mathrm{N}_{l}$, the dynamics are described by

$\dot{x}_{j}^{l}=-N_{j}^{l} x_{j}^{l}+\sum_{k \in \mathrm{N}_{j}^{l}} x_{k}^{m}+F\left(x_{j}^{l}, d\right)$

and $x_{j}^{l}$ and $x_{k}^{m}$ can be defined accordingly. By contrast, the dynamics for leaders are the same as the followers in some way, except for the impact from the target, which 
is the leaders' feature. The simulation results in the democracy strategy can be seen in Fig.1.In the process of simulation, the distances between the agents from the leader group and the follower group and the target agent are used to measure the rendezvous time indirectly, where all the distances are turned into be zero is regarded as the network rendezvous. It is not difficult to recognize that during the democracy strategy, the information flow among the different layers is fully utilized, and the enjoyment of the overall information via the shared cooperation realized win-win policy of the agents. That kinds of profit can only be available depend on the interdependency among the networks of networks. In order to elucidate the democracy in the ways of networks of networks further, the paradigm for democracy of networks of networks is illustrated as Figure 2.

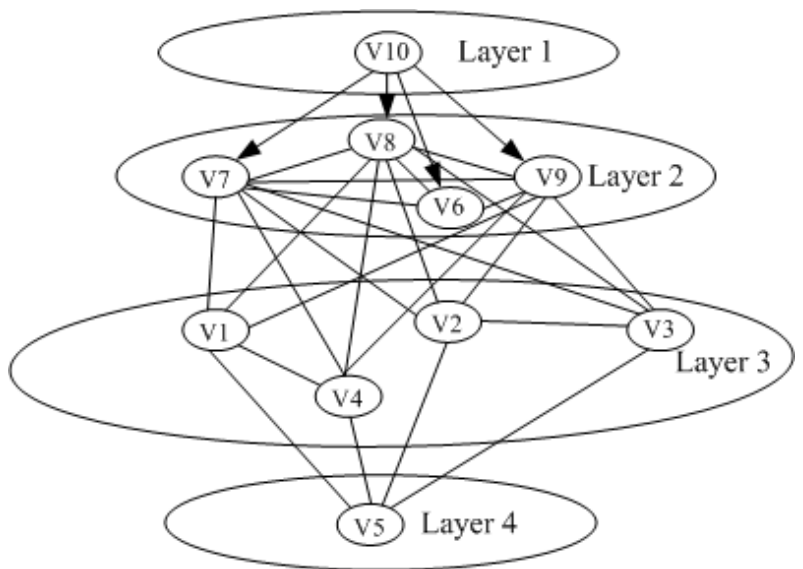

Fig. 2. The paradigm for democracy of networks of networks.

\section{Conclusion}

In this paper, according to the paradigm of networks of networks we turned the leader-follower agent systems into four different layers, and each of these layers can be equivalent to one sub-network, the connections among them can be viewed as the interdependency of the networks of networks, and we have examined and discussed the convergent characteristics of the democracy strategy. The simulation of leader-follower multi-agent network which consisted of four leaders and five followers demonstrated the potential usage of our suggested scheme.

\section{Acknowledgements}

This work is partially supported by NSFC Grant (61340041 and 61374079) and the Project-sponsored by SRF for ROCS, SEM to Yunzhong Song.

\section{References}

1. Rahmani A, Ji M, Mesbahi M, et al (2009), Controllability of multi-agent systems from a graphtheoretic perspective, SIAM Journal on Control and Optimization, 48(1) 1:162-186.

2. Olfati-Saber R, Murray R M (2004), Consensus problems in networks of agents with switching topology and timedelays, IEEE Transactions on Automatic Control, 49(9) 9:1520-1533.

3. Zavlanos M, Pappas G(2008), Distributed connectivity control of mobile networks, IEEE Transaction on Robotics, 24(6):1416-1428

4. Tove Gustavi, Dimos V Dimarogonas, Magnus Egerstedt, et $\mathrm{al}(2010)$, Sufficient conditions for connectivity maintenance and rendezvous in leader-follower networks, Automatica, 46(1):133-139.

5. Hu Jiangping, Feng Gang (2010). Distributed tracking control of leader-follower multi-agent systems under noisy measurement, Automatica, 46(8):1382-1387.

6. Ni Wei, Cheng Daizhan (2010), Leader-following consensus of multi-agent systems under fixed and switching topologies, Systems \& Control Letters, 59(3-4): 209-21.

7. Lu Renquan, Yu Wenwu, Lü Jinhu and Xue Anke (2014), Synchronization on complex networks of networks, IEEE Transactions on Neural Networks and Learning Systems, 25(11):2110-2118. 